\title{
Data Visualisation and Statistical Analysis Within the Decision Making Process
}

\author{
Jamie Mahoney \\ Centre for Educational Research and Development, University of Lincoln, Lincoln, UK.
}

\begin{abstract}
Keywords: Data visualisation; statistical analysis
Abstract: $\quad$ Large amounts of data are collected and stored within universities, but little is done to reuse this data to support decision making processes. This paper discusses the use of data visualisation and statistical analysis as methods of making sense of the collected data, analysing it to assess the effects of historical institutional decisions and discusses the use of such techniques to aid decision making processes.
\end{abstract}

\section{INTRODUCTION}

Within institutions such as universities, large amounts of data are produced and consumed during the course of an academic year. However, the data is not fully utilised or explored; often it is neglected, becomes useless and, in time, becomes nothing more than data dumps (Keim, 2002). Take, for instance, collections of data associated with awards (programmes of study for a degree) offered by a university. Data is collected and stored relating to the details of each award, the constituent modules (individual units of study) that make up the award, students and staff associated with the award, examinations and assessments for each award as well as numerous other data sources. Very little is done with this data, other than to repackage it into various formats for student handbooks or validation exercises, for example.

With such a large amount of data being collected and stored, manual textual exploration of the data may be insufficient in order to explore the data in a useful and meaningful manner (Keim, 2002; Gilbert and Auber, 2010). Visualisation tools and methods are progressively being used in a variety of situations, in order to explore and explain large amounts of data (Bastian et al., 2009; Bertschi et al., 2011). Through data visualisation, this large amount of data can be analysed more fully, in order to exploit the data being stored and to progress through the data-informationknowledge continuum (Masud et al., 2010), turning silos of data into useful information and from there, into knowledge. By visualising and analysing the vast amounts of data collected by institutions such as universities, the impact of previous decisions can be seen; these same principles can then be applied proactively, to visualise and assess the potential impact that certain decisions may have.

This paper documents the process of utilising data visualisation methods and the statistics associated with the visualisations in order to make sense of historical data sets. Having shown how these visualisation methods and statistics make sense of, and reflect changes in, the past, similar concepts could then be explored in order to assess the impact of possible future changes, thus improving the decision making process (Robertson, 1990).

\section{DATA VISUALISATION}

In order to show the extent to which data visualisation and analysis is useful within the institution, data was only collected from existing data sources that are available to staff in a variety of formats. Working in this manner has two benefits to the investigative process. Firstly, less time is spent working on ways to collect the data from other sources that are disparate and in formats that are difficult to work with. Secondly, this is data that is accessible to members of staff at the institution, with very little work required. By using this data, the focus remains on how data that the institution already has at its disposal can be used, rather than focusing on methods of collating disparate data sources.

\subsection{Description of Data}

Sufficient data was available through the reporting system for seven academic years (1st Sep - 31st Aug), starting with the academic year 2006 - 2007 through to 2012 - 2013. At the time of writing, reliable data 
(in sufficient quantity) was only available one year in advance. For each academic year, data was collected relating to each award (degree course) offered by the university, the modules (or units) that make up these awards, along with the faculties or departments that were responsible for the delivery of each module. With the introduction of new awards and old awards being phased out, the amount of data collected differs from year to year. The amount of data collected varies from 13,000 rows in $2006 / 07$ to 27,000 rows for $2012 / 13$, with student numbers varying between 9,600 and 11,700. Each row is representative of the relationship between one award and one module, i.e one award made up of ten modules would be represented by ten rows.

\subsection{Data Visualisation Principles}

Each of the data visualisations have been created based on principles taken from 'The Visual Display of Quantitative Information' (Tufte, 2001). Based on this, successful data visualisations should:

1. Show the data.

2. Make those viewing the visualisation think about the data, rather than the design of the visualisation or the technology used to construct it.

3. Avoid distorting the data.

4. Present a large amount of data in a small space.

5. Make large datasets coherent and easier to understand.

6. Allow and encourage the comparison of different pieces of information.

7. Allow the data to be viewed at several levels of detail (or granularity).

8. Serve a clear purpose, be that description, exploration, tabulation or decoration.

9. Allow the close integration of the visualisation with a statistical or verbal description.

\subsection{Initial Visualisations}

In order to show the scale of relationships between elements of the data, a network-graph visualisation showing relationships between modules of study was created for each of the academic years present in the data.

Within the graphs, each module delivered by the university is represented by a node. For each pair of modules that are delivered on the same award an edge is formed between the corresponding pair of nodes.
Further to this, groups of edges that represent the delivery of modules on the same course are then colour coded. Each node was also labelled with its module code identifier, in order to convey more meaning through the visualisation. By doing so, the depth of the data being represented is better reflected in the visualisation of the data. Figure 1 shows a small section of one of these visualisations.

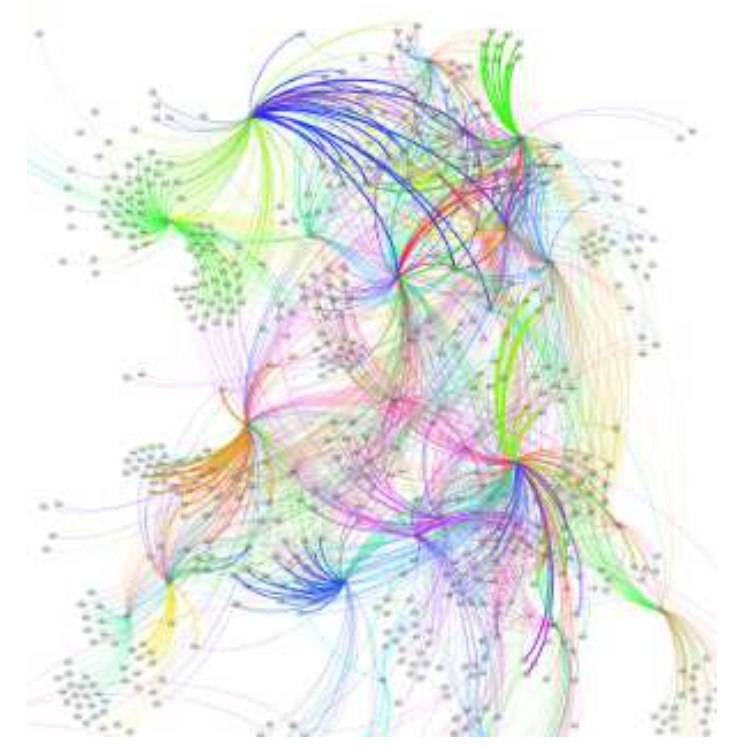

Figure 1: Part of an initial network visualization, showing the relationships between modules of study.

With the amount of data being represented in the visualisations, it becomes hard to show individual relationships between modules, but the overall pattern of relationships across the university becomes evident. Whilst these particular visualisations in their current state provide little use within decision making processes, they serve as a method of showing the scale and complexity of the data being worked with. An understanding and knowledge of the scale and complexity of the data would still be useful for those working with it and making changes to the provision of courses.

These initial visualisations did, however, provide insight into possible refinements of the data being used and also which areas to focus on in future visualisation work.

Taking a highly-granular view of the data, shows the scale of relationships across the university as a whole. In practice, modules may share more in common that just the awards they are delivered on, this is something that would need to be explored fully in further work. Whilst showing the relationships between modules, the visualisations also show the potential for adverse effects when changes are made to a module. 
It is likely that some awards rely heavily on one module for delivering a key learning outcome. Making an alteration to a module may have no consequences for four of the five awards it is delivered on, but may have serious consequences for the fifth.

Reducing the granularity of the data, it would also be useful to see links between the awards offered by the university and further to this, between departments and colleges within the institution. This would also improve the process of being able to view the visualisation as a whole - the scale of the original visualisations make it difficult to read the node labels whilst viewing the visualisation in its entirety. Creating visualisations of differing granularity would allow the user to view the data from a highly-abstracted level and 'zoom in' as required.

\subsection{Refinement of Data and Scope of Follow-Up Visualizations}

Through the creation of the exploratory data visualisations, it became apparent that a potential major audience for these data visualisations would be curriculum planners within the university. Presenting the data in this way makes it more usable; although the data was already available, data availability does not necessarily equate to data usability (Burkhard, 2005).

In terms of refinement of the data being used, only awards and modules that are active (recruiting students) and full-time have been included in the following work. By refining the data, the complexity of the main body of courses delivered at the university can be shown more clearly; the clear portrayal of complex data is a desired outcome, in order to make data visualisation a useful tool in the decision making process (Tufte, 2001).

\subsection{Visualising Relationships Between Awards}

Refining the data as discussed previously, and querying the data as to show the relationships between each award, greatly reduced the amount of nodes and edges in each network. Table 1, below, summarises the amount of edges and nodes for each network.

In a similar fashion to the original visualisations, each node is representative of an award offered at the university. Each node is labelled with the unique award code, rather than the full degree title in order to reduce clutter in the image.

Adding the magnitude of the relationships also helps to clarify the relationship between the awards, by adding detail (Tufte, 1990). These magnitudes are based solely on the amount of modules that each
Table 1: Quantities of entities represented in network visualisations.

\begin{tabular}{|c|c|c|}
\hline Academic Year & Nodes & Edges \\
\hline $2006-2007$ & 28 & 26 \\
\hline $2007-2008$ & 38 & 29 \\
\hline $2008-2009$ & 34 & 17 \\
\hline $2009-2010$ & 37 & 15 \\
\hline $2010-2011$ & 50 & 36 \\
\hline $2011-2012$ & 74 & 53 \\
\hline $2012-2013$ & 63 & 41 \\
\hline
\end{tabular}

pair of awards have in common. To add further detail to the visualisations, the nodes in the network are grouped by their owning departments, and the links between nodes colour coded in order to represent the different departments within the institution.

Figure 2 is a visualisation showing relationships between awards offered at the university, with weighted and colour-coded edges. Edges are also labelled, showing a numerical representation of the amount of links between awards.

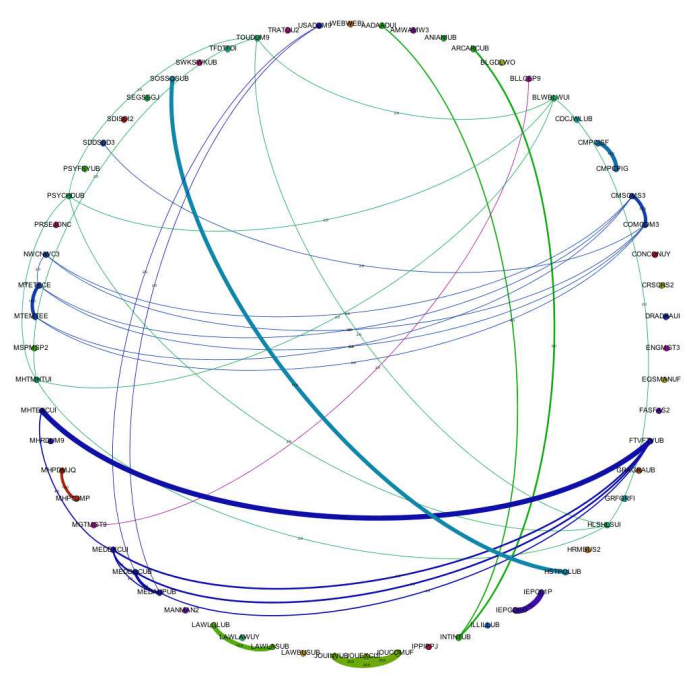

Figure 2: An example of a circular network layout showing relationships between awards.

These visualisations could be viewed in chronological order, to show the changing relationships between awards as the structure of the university and the awards that it offers to students change over time.

\section{STATISTICAL ANALYSIS OF NETWORK GRAPHS}

This section deals with the use of statistical analysis to aid the understanding of data presented in data visualisations. As the visualisations have been network 
graphs, definitions of various statistical measures are outlined and applied to this specific context and then the results explored.

\subsection{Definitions}

There are many figures and statistics that can be derived from network graphs, taking these generic definitions and applying them to the domain of the data being visualised, the following definitions can be used:

- Mean Weighted Degree of Nodes - The mean amount of modules shared between that award and other awards.

- Graph Density - The amount of connections per award when compared to the total amount of awards in the network.

- Modularity - A higher modularity suggests that awards are very highly connected with specific other awards, but have very few connections to other awards in the network. A very high modularity would suggest that a group of awards shared a lot of modules between themselves.

\subsection{Mean Weighted Degree of Nodes}

Plotting the mean weighted degree (see Figure 3), shows a changing trend over the past seven years.

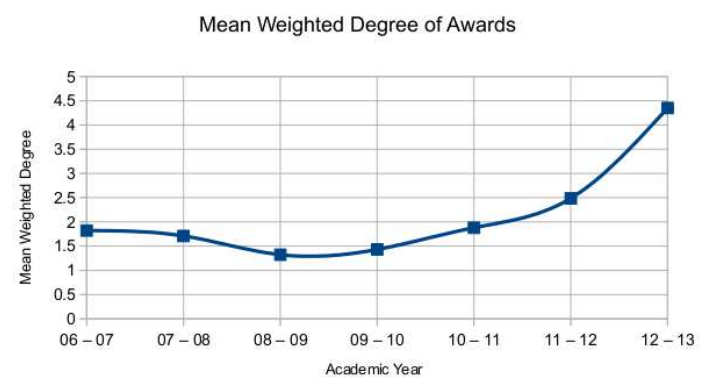

Figure 3: Mean weighted degree of awards, shown over the course of seven academic years.

Figure 3 shows a definite drop in the mean weighted degree of awards from the 2007/08 academic year to 2008/09. This shows the mean amount of links between awards dropping considerably (around 22 percent). Combining this data with information relating to changes in the university, it becomes apparent that this drop in the mean weighted degree occurs when the university began to reorganise the delivery of degree awards. Prior to 2007, an academic year consisted of 120 points of study broken down into 12 or 24 point modules, depending on whether the module was delivered over a single or two semesters. From 2007, a new system was introduced, in a phased process, where new students would take 120 points of study, broken down into 15 and 30 points modules. As a standard, full-time degree takes three years to complete, there was a two year period where both forms of study were being undertaken by different cohorts of students.

From the highest point on the first portion of the graph, to this point in the new weighting, there is an increase of over 36 percent in the amount of joins between awards offered at the university. This shows that (assuming an increase in mean weighted degree is good in terms of curriculum design) the provision has been improved through the restructuring of awards during the alteration of module weightings.

\subsection{Network Modularity}

Another statistical measure that appears to have been heavily influenced by changes in the structure of the university is the modularity of the awards, shown in Figure 4.

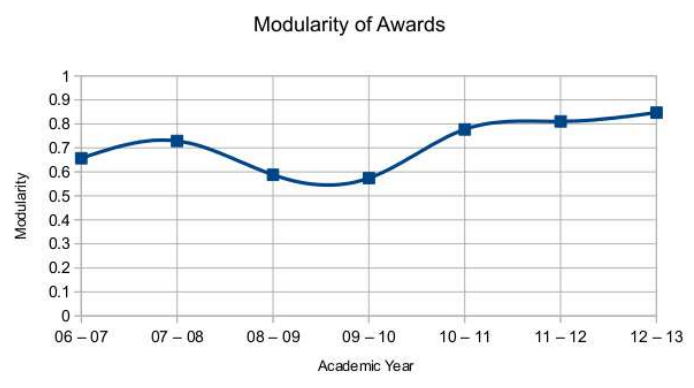

Figure 4: Modularity of awards, shown over the course of seven academic years.

A high modularity value shows that the awards are forming into self-contained clusters, with connections predominantly within the cluster, and few connections to other clusters or outliers. As shown in Figure 4, the modularity of awards in 2006 - 2007 was relatively high, this figure continues to rise, before dropping for two consecutive years as the weighting of modules at the university goes through a period of change. As the change is fully implemented, the modularity rises significantly and continues to rise from 2010 2011 through to 2012 - 2013. This would suggest that (though not necessarily the case) either by design or good fortune, the awards offered at the university are starting to form into more easily-identified selfcontained groups, perhaps areas of specialism. This point is interesting to note, as the institution has gone through a period of change, in terms of how departments within the university are organised.

There is, however, an issue with using network modularity as a standalone indicator, primarily due 
to there being a 'resolution threshold', beneath which smaller clusterings of nodes become 'invisible' (Fortunato and Barthélemy, 2007; Kumpula et al., 2007). Combining multiple statistical indicators in order to get a richer and more reliable indication of the state of the network is discussed in a later sub-section.

\subsection{Network Graph Density}

The density of the network shows the mean amount of connections between nodes as a proportion of the maximum amount of connections available, with values ranging between 0 and 1 . A value of 1 , or a 'complete graph' shows that each node in the network is connected to every other node; with a value of 0 showing no connections at all between nodes.

In this context, neither a value of 0 or 1 would be desirable, further work would be required in order to determine, if possible, an ideal value of range of values.

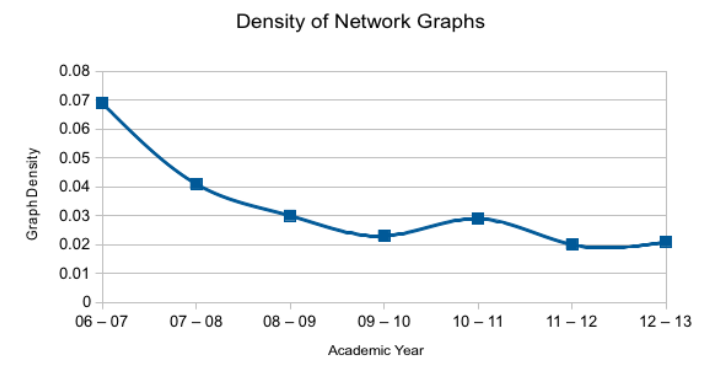

Figure 5: The density of award networks, shown over the course of seven academic years.

Figure 5 shows a dramatic decrease in graph density from the academic year 2006 - 2007 to 2007-2008 before an eventual decrease over the next five academic years. It has become evident that this statistical measure is perhaps not best suited for use as an indicator when considering the relationships between awards, but may be better suited to networks where relationships are more extensive, i.e. the original visualisations showing module relationships. The introduction of one or two new awards would have a fairly substantial impact on the density of the awards graph and may misrepresent the true effects of introducing the new awards.

\subsection{Combining Statistical Measures}

Whilst the statistical measures discussed previously are useful indicators of the effects of changes to the provision of degrees and the structure of the institution, combining the measures may help to provide a fuller understanding of the potential impact of decisions made in the future.
Take for instance, a situation in which the impact of making decision $\mathrm{X}$ is being assessed. By showing the altered data in network form and analysing the statistics, the impact can be judged. For example, if the changes were to result in an increase in modularity, yet a decrease in the mean weighted degree, then this would suggest that, whilst distinct groups of awards or modules were being formed, they are quite likely to be forming small, highly separated clusters. This could then be used to help determine if the course of action being decided upon may produce positive and desired results.

\subsection{Evaluation of Statistical Analysis}

A selection of statistical measures of network graphs have been highlighted and contextualised. These have been explored in more detail, and changes in the institution used to explain clear changes in the trends being shown in the statistics. This demonstrates that these metrics can be used to show the impact that changes to the institution can have on these figures. This suggests that these same principles can be used proactively in the decision-making process to show the resulting impact of various potential decisions.

\section{CONCLUSIONS AND FURTHER WORK}

Through the process of exploring large and complex data sets, it has been shown that data visualisations are a useful tool in improving understanding of data. These initial exploratory data visualisations also prove useful in helping to determine potential uses and users of data visualisations in later work.

By refining the data in order to focus on the assumed requirements of those expected to use the visualisations, the scale of data being presented is reduced somewhat, resulting in clearer visualisations. However, this is not always beneficial as some statistical measures become distorted or almost useless when used on data with a low level of granularity.

The full extent of relationships between modules and awards would have to be explored in order to show users a true representation. It would be inappropriate to use representations of incomplete data to aid in decisions. By collecting more data relating to each aspect of these awards and modules that can be used to link them, an application can be built that allows the data to be interacted with during the decision making process, showing the impact of potential alterations to individual elements. This application would allow the 
user to view the data being presented at varying levels of granularity, similar to the ways in which some of the data has been presented in this paper. By doing so and allowing the user to change the structure of the data that is presented to them, the various effects of making seemingly small changes to the provision of education within the university can be easily displayed. By doing so, the decision making process could be made more efficient and effective. A casestudy approach would allow the evaluation of both the proposed interactive visualisations and the statistical analysis. The monitoring and assessment of ongoing business processes plays an important role in the continuity of organisations (Rinderle et al., 2006); the integration of data visualisation and statistical analysis into the decision making process would help the process of reviewing and assessing the impact of decisions made within the institution.

\section{ACKNOWLEDGMENTS}

The work described in this paper was carried out as part of a JISC-funded project, under the 'Course Data : Making the Most of Course Information' funding call.

\section{REFERENCES}

Bastian, M., Heymann, S., and Jacomy, M. (2009). Gephi: An open source software for exploring and manipulating networks. International AAAI Conference on Weblogs and Social Media.

Bertschi, S., Bresciani, S., Crawford, T., R, G., Kienreich, W., Lindner, M., Sabol, V., and Moere, A. (2011). What is knowledge visualization? perspectives on an emerging discipline. 15th International Conference on Information Visualization, pages 329-336.

Burkhard, R. (2005). Knowledge Visualization: The Use of Complementary Visual Representations for the Transfer of Knowledge - A Model, a Framework, and Four New Approaches. $\mathrm{PhD}$ thesis, Swiss Federal Institute of Technology (ETH Zurich).

Fortunato, S. and Barthélemy, M. (2007). Resolution limit in community detection. Proceedings of the National Academy of Sciences of the United States of America, 104(1):36-41.

Gilbert, F. and Auber, D. (2010). From database to graph visualization. 14th International Conference Information Visualization, pages 128-133.
Keim, D. (2002). Information visualization and visual data mining. IEEE Transactions on Visualization and Computer Graphics, 7(1):1-8.

Kumpula, J., Saramäki, J., Kaski, K., and Kertesz, J. (2007). Limited resolution in complex network community detection with potts model approach. The European Physics Journal B, 56(1):41-45.

Masud, L., Valsecchi, F., and Ciuccarellia, P. (2010). From data to knowledge - visualizations as transformation processes within the data - information - knowledge continuum. 14th International Conference Information Visualization, pages $445-499$.

Rinderle, S., Bobrik, R., Reichert, M., and Bauer, T. (2006). Business process visualizaiton - use cases, challenges, solutions. Proceedings of the Eighth International Conference on Enterprise Information Systems (ICEIS'06): Information System Analysis and Specification, pages $204-$ 211.

Robertson, P. (1990). A methodology for scientific data visualisation : Choosing representations based on a natural scene paradigm. Proceedings of the First IEEE Conference on Visulaisation, pages 114-123.

Tufte, E. R. (1990). Envisioning Information. Graphic Press USA.

Tufte, E. R. (2001). The Visual Display of Quantitative Information. Graphic Press USA, 2nd edition. 